\title{
Exogenous S-Methylmethionine Alleviates Salinity Stress by Modulation of Physiological Processes in Canola (Brassica napus)
}

\author{
Laszlo Fodorpataki ${ }^{1,2^{*}}$, Katalin Molnar ${ }^{3}$, Bernat Tompa ${ }^{1}$ and Csaba Bartha ${ }^{3}$ \\ ${ }^{1}$ Hungarian Department of Biology and Ecology, Babes-Bolyai University, RO-400084 Cluj-Napoca, Romania \\ ${ }^{2}$ Laboratory of Plant and Microbe Biotechnologies and Environmental Interactions, Centre of Systemic Biology, Biodiversity \\ and Bioresources, Babes-Bolyai University, RO-400084 Cluj-Napoca, Romania \\ ${ }^{3}$ Department of Horticulture, Sapientia Hungarian University of Transylvania, RO-540485 Targu Mures, Romania
}

*For correspondence: lfodorp@gmail.com

Received 07 May 2020; Accepted 27 August 2020; Published 10 December 2020

\begin{abstract}
Canola is a moderately salt tolerant plant, high salinity inhibits germination of seeds, vegetative growth of young plantlets, and reduces biomass production. This study investigated the effects of priming with $1 \mathrm{~m} M \mathrm{~S}$-methylmethionine (SMM) on germination, leaf gas exchange, induced chlorophyll fluorescence, photosynthetic pigment content and membrane damage through lipid peroxidation by exposing canola (Brassica napus L. cv. Cindi) plants to moderate and severe salt stress (induced by $60 \mathrm{mM}$ and $120 \mathrm{mM} \mathrm{NaCl}$ ) for different periods. Priming with SMM alleviated the reduction of net photosynthetic rate, the effective quantum efficiency and efficiency of excitation energy capture by open photosystem II reaction centers, chlorophylls to carotenoids ratio, enhanced water use efficiency and contributed to reduction of oxidative membrane damage in fully developed young leaves. Delay and inhibition of seed germination by salt stress were significantly reduced by SMM, as well as the non-photochemical quenching of the singlet excited state of chlorophyll $a$, suggesting a more efficient protection against hyperosmotic stress, ionic toxicity and associated oxidative stress in primed plants exposed to high salinity. This first assay of SMM as a priming agent for canola plants under high salinity contributes to a better understanding of the mode of action of this natural, plant-derived bioactive compound and the optimization of canola cultivation under the adverse growth conditions caused by salt stress. (C) 2021 Friends Science Publishers
\end{abstract}

Keywords: Photosynthetic performance; Priming; Salt tolerance; Stomatal conductance

\section{Introduction}

Priming of crop plants, at developmental stages sensitive to adverse environmental conditions, proved to be a costeffective procedure which improves stress tolerance and enhances plant production without any genetic manipulation (Tabasssum et al. 2017; Farooq et al. 2018). Defense mechanisms may be accelerated or intensified by pretreatment with different substances which act as stressors, signaling molecules or bioregulators (Yang and Lu 2005; Farooq et al. 2009, 2013, 2017, 2019; Sivaci et al. 2014; Kaya et al. 2015). Even biological priming agents (rootassociated microbial communities, e.g., arbuscular mycorrhizal fungi) were shown to be effective in crop stress management (Balestrini et al. 2018; Rehman et al. 2018). Because short treatments with low quantities of priming agents have multiple and long-lasting physiological effects during hardening, the number of naturally occurring substances with antistress potential is increasing, and novel agricultural practices are developed in order to improve the bioproductive potential of several crop plants in extended regions where climatic changes cause more severe abiotic stress (Bajwa et al. 2018; Mahboob et al. 2019). For some priming agents it was demonstrated that they induce transcriptional modifications, post-translational protein modifications and epigenetic modifications, which may confer prolonged protection during stress exposure. Downregulation of generation of reactive oxygen-nitrogen-sulfur species and up-regulation of aquaporin and dehydrin expression levels were also attributed to certain priming agents used to counteract hyperosmotic stress (Vannier et al. 2015; Antoniou et al. 2016; Savvides et al. 2016).

S-methylmethionine (SMM) is a universal metabolite of flowering plants, being produced from the amino acid methionine. Its physiological functions are highly unknown, but there is evidence about its role as a methylation agent, and it is supposed to be implied in the regulation Sadenosylmethionine (SAM) level in plant cells (McRorie et al. 1954; Ranocha et al. 2001). It down-regulates monoamine oxidase activity and increases RNA level, which indicates its influence on gene expression. In some plant species, it is the precursor for the biosynthesis of dimethyl-

To cite this paper: Fodorpataki L, K Molnar, B Tompa, C Bartha (2021). Exogenous S-methylmethionine alleviates salinity stress by modulation of physiological processes in canola (Brassica napus). Intl J Agric Biol 25:11-19 
sulfoniopropionate, which is an effective osmoregulator during drought and cold stress. It is easily translocated both through the xylem and phloem sap, its highest amounts being detected in young leaves (Ranocha et al. 2001; Iqbal et al. 2013). It is also known as vitamin U, although in the strict sense of the term it cannot be considered a true vitamin, because even if it has beneficial effects on several metabolic processes, it was not proved to be an essential organic micronutrient for humans (Patel and Prajapati 2012). Previous studies demonstrated that addition of micromolar or millimolar concentrations of SMM to the nutrient solution contributed to the tolerance of hypoxia, of mosaic virus infection, of heavy metal toxicity and of low temperature in certain crop species (Paldi et al. 2014). Its utility as a priming agent was reported only in lettuce plants exposed to cold stress and to high salinity, where it also increased the healthpromoting properties of fresh leaves (Fodorpataki et al. 2016, 2019). All these studies lead to the idea that exogenous supplementation of SMM may promote plants' growth and development under environmental stress conditions.

Salt stress is one of the most widespread abiotic factors limiting the growth and the productivity of crops, and it affects more and more arable lands as climatic changes related to rising temperatures intensify the evaporation of soil water, and irrigation techniques also contribute to elevated soil salinity (Hollington and Steele 2007; Nayidu et al. 2013). Most of the crop plant species and cultivars are salt sensitive, with a certain degree of tolerance which changes during developmental stages and in accordance with cultivation practices (Zhu 2001). In plants, high salinity stress has a rapid phase in which it causes imbalance in the water homeostasis, leading to turgor loss, stomatal closure and growth inhibition. These effects can be reversed mainly by osmoregulation and modulation of stomatal gas exchange processes (Downton et al. 1990; Yang and Lu 2005; Shabhaz et al. 2013; Mahboob et al. 2019). The long-term phase of salt stress is due to accumulation of excessive amounts of sodium and chloride ions in the plant cells, resulting in chemical toxicity caused by an imbalance in ion homeostasis, because sodium ions compete with potassium ions and also have an antagonistic interrelation with calcium ions. This toxic effect may be counteracted by compartmentation of sodium ions into the vacuoles, in the cell wall spaces or in the xylem sap, and partly by sequestration of salt in the root system (Blumwald 2000; Demetriou et al. 2007; Rasheed et al. 2014). A sideeffect of high salinity is accumulation of reactive oxygen species, which exert an oxidative stress. This may be annihilated by up-regulation of antioxidative defense mechanisms, involving increased production of nonenzymatic antioxidants and enhanced catalytic activity of antioxidative enzymes (Tang and Newton 2005; Kaya et al. 2015; Srivastava et al. 2015).

Present understanding on how S-methylmethionine acts as a priming agent in plants is very limited, and little information is available about the influence of exogenous
SMM on seed germination, leaf gas exchange, photosynthetic parameters and membrane stability of canola exposed to salt stress. Therefore, the objective of this study is to define effects of pre-treatment with SMM on physiological processes in canola plants. This aims to improve understanding of the mechanisms regarding the alleviation of salinity stress and to apply priming with SMM for optimization of canola growth, photosynthetic performance and salt stress tolerance. Our hypothesis is that priming with SMM solution can enhance salt tolerance of canola by attenuating negative effects of high salinity on photochemical processes of photosynthesis, on gas exchange parameters and on other physiological traits related to improved crop production and to acclimation to salt toxicity.

\section{Materials and Methods}

\section{Experimental details}

The experiments were carried out with canola (Brassica napus L. ssp. oleifera) plants belonging to the "Cindi" cultivar. This was selected from ten frequently grown cultivars (Avenir, Chalki, Chelsi, Cindi, Facti, Intense, Nodari, Kodiak, Triangle and Tripti, the seeds being purchased from Caussade Semences, Alcedo and KWS), based on previous studies which established that Cindi is the most salt-sensitive. For germination, uniform sized healthy seeds, 100 in three repetitions for each treatment setup, were selected and sterilized in $3 \%(\mathrm{w} / \mathrm{v})$ sodium hypochlorite solution for $10 \mathrm{~min}$ and washed twice with distilled water (Abdolahi et al. 2012). For the other experiments, sterilized seeds were pre-hydrated for $12 \mathrm{~h}$ with distilled water and germinated in Linhard vessels at $20^{\circ} \mathrm{C}$. After one week, plantlets with similar size, ten for each experimental variant (i.e., a total of 60 plants), were planted separately in pots with perlite, watered regularly with half-strength Hoagland nutrient solution (Hoagland and Arnon 1950) and grown for two weeks in an environmental chamber (Sanyo MLR$351 \mathrm{H})$ under a photosynthetically active photon flux density of $540 \mu \mathrm{mol} \mathrm{m} \mathrm{m}^{-2}$ for a daily photoperiod of $14 \mathrm{~h}$, at $22^{\circ} \mathrm{C}$ during the light period and $18^{\circ} \mathrm{C}$ in the dark period, the relative air humidity being maintained at $60 \%$ (Zamani et al. 2010; Fiebelkorn and Rahman 2016). After two weeks of development, three series of 10 plants were primed by spraying on their aboveground shoot (leaves and stem) an aqueous solution of $1 \mathrm{~m} M \quad \mathrm{~S}$-methylmethionine. This concentration was selected based on previous experiments, considering that quantities used once as a pre-treatment are usually higher than those established for continuous treatment during the experimental period (Paldi et al. 2014; Fodorpataki et al. 2016). $12 \mathrm{~h}$ after the pre-treatment with SMM, several series of ten plantlets each, primed and not primed, were exposed for 4 days to moderate and severe salt stress, induced with $60 \mathrm{~m} M$ and $120 \mathrm{~m} M$ of $\mathrm{NaCl}$ dissolved in the Hoagland nutrient solution used to irrigate the perlite in the pots, once in two days at 8 a.m. Thus, six experimental 
variants were set up, each with ten plantlets: the control provided with basic Hoagland solution, a series pre-treated with $1 \mathrm{~m} M$ SMM but not exposed to subsequent salt stress, a series exposed to $60 \mathrm{mM} \mathrm{NaCl}$, one to $120 \mathrm{~m} M \mathrm{NaCl}$ and two series of ten plants each, primed with $1 \mathrm{~m} M$ SMM and then submitted to $60 \mathrm{~m} M \mathrm{NaCl}$ and $120 \mathrm{~m} M \mathrm{NaCl}$, respectively. Growth conditions during the treatments were similar with those created for development of seedlings after germination.

\section{Measurements}

Germination dynamics: Surface sterilized seeds, three repetitions of 100 seeds each, were soaked for $12 \mathrm{~h}$ in different aqueous solutions according to experimental variants: control and non-primed lots were immersed in distilled water, while primed seeds were covered by an aqueous solution of $1 \mathrm{~m} M \mathrm{~S}$-methylmethionine. After $12 \mathrm{~h}$, all of the seed lots were put at equal distances from each other in Linhard germination vessels, on double-layered filter paper thoroughly imbibed with distilled water for control lots and for those receiving only SMM pre-treatment, and with $60 \mathrm{~m} M$ and $120 \mathrm{~m} M \mathrm{NaCl}$ solutions, according to the two different salt treatments. Some salt-stressed seed lots were not pre-treated with SMM, while the others were primed for $12 \mathrm{~h}$ with $1 \mathrm{~m} M$ SMM. The seeds were kept at $20^{\circ} \mathrm{C}, 60 \%$ relative air humidity and $220 \mu \mathrm{mol} \mathrm{m} \mathrm{m}^{-2} \mathrm{~s}^{-1}$ photon flux density $10 \mathrm{~h}$ a day, for 8 days, the filter papers being kept saturated with the treatment solutions. The germinated seeds (with the radicle emerged through the seed tegument) were recorded every second day during the morning hours.

Chlorophyll fluorescence: Conventional and pulse amplification modulated parameters of induced chlorophyll fluorescence were measured on the fourth emerging leaf of each plant, with an FMS-2 type fluorometer (Hansatech). On dark-adapted leaves, just before the onset of the light period, the ground fluorescence $\left(\mathrm{F}_{0}\right)$ was measured by illuminating the leaves with a dim red light flash $(0.1 \mu \mathrm{mol}$ $\left.\mathrm{m}^{-2} \mathrm{~s}^{-1}\right)$, while maximum fluorescence $\left(\mathrm{F}_{\mathrm{m}}\right)$ was recorded during a subsequent saturating light pulse $(10000 \mu \mathrm{mol} \mathrm{m}$ $\mathrm{s}^{-1}$ for $0.5 \mathrm{~s}$ ). The leaves were then continuously illuminated with actinic light of $800 \mu \mathrm{mol} \mathrm{m} \mathrm{m}^{-2}$, in order to determine the steady state fluorescence $\left(\mathrm{F}_{\mathrm{s}}\right)$. A second saturating white light flash was imposed to record the modulated maximum fluorescence in the light-adapted state $\left(\mathrm{F}_{\mathrm{m}}{ }^{\prime}\right)$, then the actinic light was turned off, and the modulated minimal fluorescence in the light-adapted state $\left(\mathrm{F}_{0}{ }^{\prime}\right)$ was measured by illuminating the leaf with far-red light $\left(30 \mu \mathrm{mol} \mathrm{m} \mathrm{m}^{-2}\right.$ for $3 \mathrm{~s}$ ). From the recorded values, the following parameters were calculated and selected for interpretation: (1) the effective quantum efficiency of photosystem II (PSII): ФPSII $=\left(\mathrm{F}_{\mathrm{m}}{ }^{\prime}-\mathrm{F}_{\mathrm{s}}\right) / \mathrm{F}_{\mathrm{m}}$, , (2) the efficiency of excitation energy capture by open PSII reaction centers: $F_{v}{ }^{\prime} / F_{m}{ }^{\prime}=\left(F_{m}\right.$, $\left.-\mathrm{F}_{0}{ }^{\prime}\right) / \mathrm{F}_{\mathrm{m}}{ }^{\prime}$, and (3) the non-photochemical quenching of the singlet excited state of chlorophyll $a$ : $\mathrm{NPQ}=\left(\mathrm{F}_{\mathrm{m}}-\mathrm{F}_{\mathrm{m}}{ }^{\prime}\right) / \mathrm{F}_{\mathrm{m}}$ ', (Kooten and Snel 1990; Maxwell and Johnson 2000; Xia et al. 2004; Kalaji et al. 2018).

Gas exchange parameters: Gas exchange parameters were monitored at the start of salt treatment, on the second and on the fourth day of exposure to salt stress, before noon, on the abaxial side of the fourth fully expanded leaf from the base of stem, with a Ciras-2 type leaf gas exchange meter (PP Systems). In the measurement chamber leaf temperature was maintained at $22^{\circ} \mathrm{C}, \mathrm{CO}_{2}$ concentration at $400 \mu \mathrm{mol}$ $\mathrm{mol}^{-1}$, the relative air humidity at $60 \%$ and light intensity at $540 \mu \mathrm{mol} \mathrm{m} \mathrm{m}^{-2} \mathrm{~s}^{-1}$ photosynthetic photon flux density. Net photosynthetic carbon dioxide assimilation rate (Pn), stomatal conductance to water vapor $\left(\mathrm{g}_{\mathrm{s}}\right)$ and transpiration rate $(\mathrm{Tr})$ of leaves were measured, while the water use efficiency (WUE) was calculated as the net photosynthetic carbon dioxide assimilation rate to transpiration rate $(\mathrm{Pn} / \mathrm{Tr})$ ratio (Nieva et al. 1999; Zhang et al. 2009; Ceusters et al. 2019). Gas exchange and chlorophyll fluorescence measurements were made, separately, on the same portion of the leaf blades, between morning and noon.

Quantification of photosynthetic pigments: Photosynthetic pigments were extracted from the fourth fully expanded leaf from the base of the stem, from the same leaf blade region on which gas exchange and chlorophyll fluorescence measurements were performed in vivo straight before pigment determination, on the fourth day of salt exposure, around noon. Extracts were obtained from $0.25 \mathrm{~g}$ of leaves (fresh weight) finely homogenized in $5 \mathrm{~mL} 80 \%(\mathrm{v} / \mathrm{v})$ acetone, then the supernatant resulting from centrifugation for $10 \mathrm{~min}$ at $4000 \mathrm{~g}$ and $4{ }^{\circ} \mathrm{C}$ was used for absorbance measurements, using a UV-Vis spectrophotometer (Jasco). Concentrations of chlorophyll $a, b$ and carotenoids were calculated according to Wellburn (1994), then total chlorophylls to total carotenoids ratio was computed.

Membrane lipid peroxidation assay: Oxidative damage of membranes was evaluated on the basis of formation of malondialdehyde (MDA) and other thiobarbituric acidreactive substances (TBARS) due to peroxidation of unsaturated fatty acids in membrane lipids (Baryla et al. 2000). $0.5 \mathrm{~g}$ fresh leaf samples, from the same leaves on which gas exchange measurements, chlorophyll fluorescence determinations and photosynthetic pigment quantifications were made, were homogenized with $5 \mathrm{~mL}$ of $0.1 \%(\mathrm{w} / \mathrm{v})$ trichloroacetic acid (TCA) solution in a prechilled mortar, the homogenate was centrifuged at $15000 \mathrm{~g}$ for $15 \mathrm{~min}$, then $2 \mathrm{~mL}$ of the supernatant was transferred in a test tube and $4 \mathrm{~mL}$ of $10 \%(\mathrm{w} / \mathrm{v})$ TCA with $0.5 \%(\mathrm{w} / \mathrm{v}) 2$ thiobarbituric acid (TBA) were added. The mixture was heated at $95^{\circ} \mathrm{C}$ for $30 \mathrm{~min}$ and then quickly cooled in an ice bath. The cooled solution was centrifuged at $10000 \mathrm{~g}$ for 5 min, and the absorbance of the supernatant was measured at $532 \mathrm{~nm}$ and $600 \mathrm{~nm}$. The absorbance at $600 \mathrm{~nm}$ (due mainly to interference of anthocyanin pigments) was deducted from the value obtained at $532 \mathrm{~nm}$. MDA concentration of samples was calculated using the extinction coefficient of $155 \mathrm{~m} M^{-1} \mathrm{~cm}^{-1}$ (Tang and Newton 2005; Jambunathan 2010). 


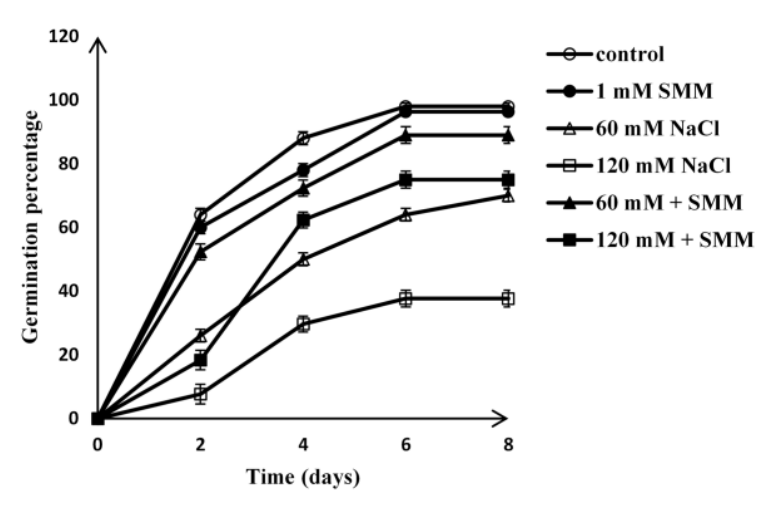

Fig. 1: Effects of $\mathrm{NaCl}$ and/or priming with S-methylmethionine (SMM) on germination of canola seeds. Vertical bars represent \pm SE from means

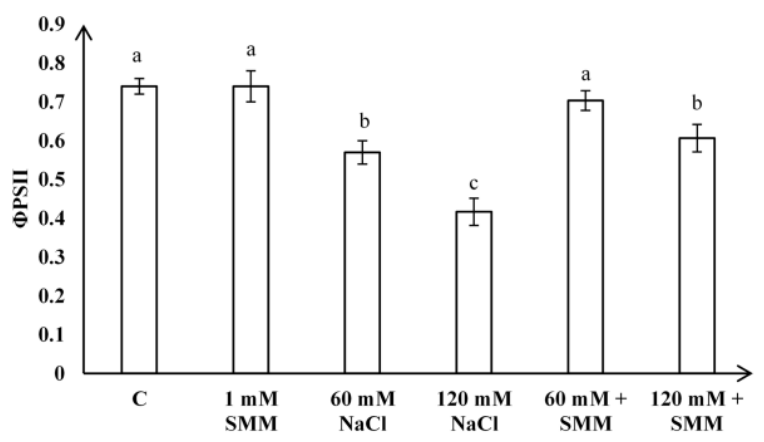

Fig. 2: Effective quantum efficiency of photosystem II (ФPSII) in leaves of canola plants exposed for four days to high salinity, with or without priming with S-methylmethionine (SMM). C is the control without priming and salt treatment, $1 \mathrm{~m} M$ SMM refers to plants primed with SMM but not exposed subsequently to $\mathrm{NaCl}$ stress. Bars represent \pm SE from means $(n=10)$, different letters above the columns indicate significant differences at $P<0.05$

\section{Statistical analyses}

All the experiments were performed with ten plants for every treatment type, except for germination, in which case experiments with 100 seeds for each treatment were conducted in triplicate. Measurements of physiological parameters were repeated three times. Data analysis was performed with the $\mathrm{R}$ statistical package ( $\mathrm{R}$ Core Team 2019), using the Shapiro-Wilk test for normality and Bartlett's test for homogeneity of variances. Data were represented as the mean \pm standard error (SE). The significant differences were determined by the one-way ANOVA test and the post-hoc Tukey HSD test. Differences were considered significant at $P<0.05$.

\section{Results}

\section{Germination}

Priming for $12 \mathrm{~h}$ with $1 \mathrm{~m} M$ S-methylmethionine did not cause significant changes in the dynamics of canola seed germination, but it considerably alleviated the delaying and inhibitory effects of high salinity (Fig. 1). Control seed lots had a germination percentage higher than $60 \%$ after two days, higher than $80 \%$ after four days and a maximum of around $98 \%$ reached until the sixth day. Pre-treatment with SMM did not result in significant changes, except for a very moderate, but statistically significant decrease of germination percentage on the fourth day, which recovered by the sixth day. Salt stress delayed germination of most seeds and caused a lowered final germination percentage. Under moderate salt stress $(60 \mathrm{mM} \mathrm{NaCl})$ less than $30 \%$ of seeds germinated until the second day and around $60 \%$ germinated until the end of the 8 days period. When severe salt stress was applied with $120 \mathrm{mM} \mathrm{NaCl}$, the final germination percentage was lower than $40 \%$, and during the first two days less than $10 \%$ of seeds could germinate. These impairments caused by high salinity were significantly counterbalanced by priming with SMM: germination percentage of seeds exposed to $60 \mathrm{mM} \mathrm{NaCl}$ was increased from around $60 \%$ to about $80 \%$ in six days, and on the second day more than $50 \%$ of seeds managed to germinate (instead of an average of 26\%). In the case of more severe salt stress, the benefic influence of seed priming with SMM was even more pronounced: the delay in germination was highly recovered between the second and the fourth day (an average of $62 \%$ instead of $29 \%$ in nonprimed seed lots), and the final germination percentage was increased from $38 \pm 2$ to $74 \pm 3 \%$.

\section{Photosynthetic performance}

Effective quantum efficiency of photosystem II (ФPSII) was not influenced by priming with $1 \mathrm{~m} M$ SMM in plants which were not exposed to salt stress. ФPSII of canola plants grown for four days in the presence of high salinity was markedly reduced. This reduction was proportional with salt concentration, and it was significantly alleviated by pretreatment with $1 \mathrm{~m} M$ SMM. In plants exposed to $60 \mathrm{~m} M$ $\mathrm{NaCl}$, priming with SMM totally annihilated the lowering effect of moderate salt stress, while in the plants grown under a more severe salt stress $(120 \mathrm{mM} \mathrm{NaCl})$, pretreatment resulted in a partial compensation for the inhibitory effect (Fig. 2). The efficiency of excitation energy capture by open PSII reaction centers (Fv'/Fm') of the plants treated with $\mathrm{NaCl}$ was reduced with time, this reduction being more pronounced in the presence of 120 $\mathrm{m} M \mathrm{NaCl}$. Priming with S-methylmethionine alleviated this reduction, and in the case of plants exposed to $120 \mathrm{mM}$ $\mathrm{NaCl}$ maintained a constant value of this photosynthetic parameter. SMM had no significant influence on $\mathrm{Fv}^{\prime} / \mathrm{Fm}^{\prime}$ in plants not exposed to high salinity (Fig. 3a). Nonphotochemical chlorophyll fluorescence quenching (NPQ) was shown to have different characteristics from the other photosynthetic parameters determined in vivo in canola leaves, namely, it continuously increased during the 


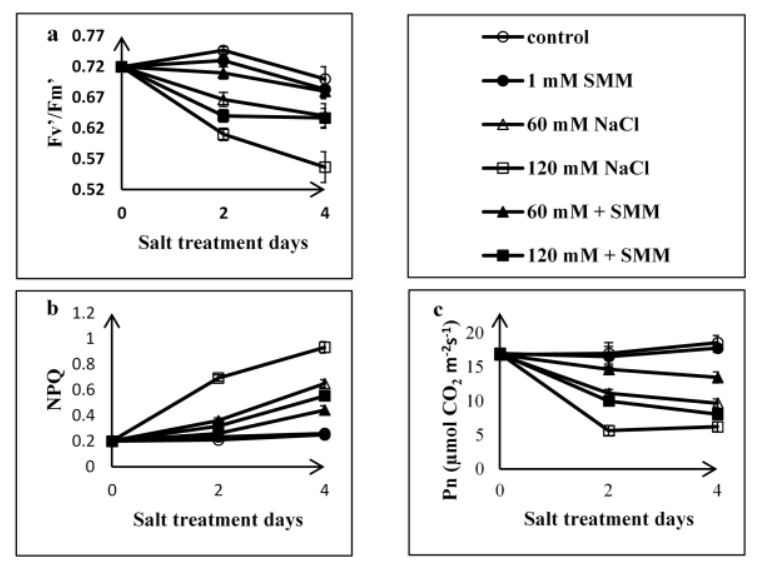

Fig. 3: Effects of $\mathrm{NaCl}$ and/or priming with S-methylmethionine (SMM) on the efficiency of excitation energy capture by open photosystem II reaction centers (Fv'/Fm') (a), on the nonphotochemical quenching of chlorophyll $a$ fluorescence (NPQ) (b), and on the net photosynthetic carbon dioxide assimilation rate (Pn) (c) in leaves of canola plants. Bars represent \pm SE from means $(\mathrm{n}=10)$

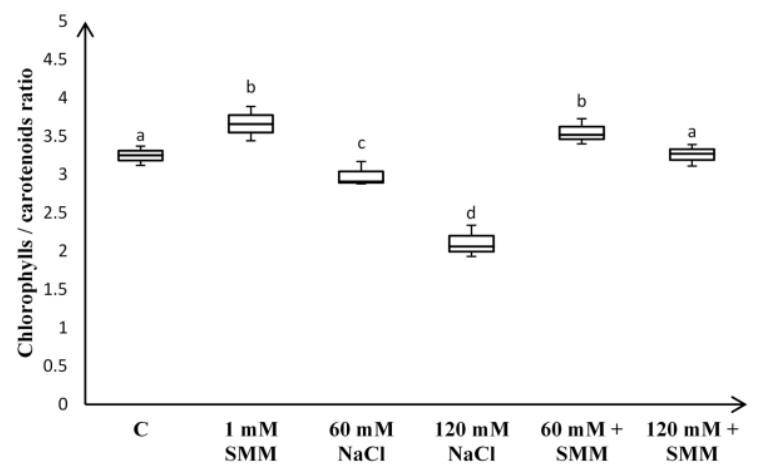

Fig. 4: Chlorophylls to carotenoids pigment ratio in leaves of canola plants developed for four days under salinity, with or without priming with S-methylmethionine (SMM)

experiment in all plants exposed to salt stress, irrespective of priming treatment. The NPQ of plants exposed to $\mathrm{NaCl}$ was enhanced during the latter part of the experimental period, but this enhancement was significantly attenuated in SMMprimed plants, especially in the case of exposure to $120 \mathrm{mM}$ $\mathrm{NaCl}$. Pre-treatment with SMM in unstressed plants had no influence on NPQ (Fig. 3b). Net photosynthetic rate (Pn), measured through carbon dioxide uptake of leaves, was reduced by salt treatment, the reduction being more pronounced in the first half of the exposure period. This reduction was alleviated by priming with $1 \mathrm{~m} M$ SMM at both $\mathrm{NaCl}$ concentrations, but the values remained under the ones registered for control and only SMM pre-treated canola plants. On the fourth day of salt treatment, the alleviation was more pronounced in plants exposed to $60 \mathrm{mM} \mathrm{NaCl}$, in comparison with the ones grown in the presence of $120 \mathrm{mM}$ $\mathrm{NaCl}$ (Fig. 3c).

\section{Photosynthetic pigment ratio}

The ratio between total chlorophylls $(a+b)$ and total carotenoid pigment content of fully developed canola leaf blades in the period of midday was around 3.25 in control plants and pre-treatment with $1 \mathrm{~m} M$ S-methylmethionine induced a moderate, but statistically significant increment of this ratio. Salt treatment for four days reduced the chlorophylls to carotenoids ratio proportionally with the salt concentration (to a value around 2.0 in the presence of 120 $\mathrm{m} M \mathrm{NaCl}$ ). This reduction was completely offset by priming with SMM of salt-stressed plants, in which case the ratio remained similar to control or was even higher in the primed plants exposed to $60 \mathrm{mM} \mathrm{NaCl}$ (Fig. 4).

\section{Water use efficiency}

Under non-salt stress condition, spraying of an aqueous solution of $1 \mathrm{mM}$ S-methylmethionine had no significant effect on water use efficiency of leaves during the subsequent four days, and maintained its value around 0.002 . Moderate salinity slightly increased WUE until the second day of treatment and this value was maintained during the next two days. Exposure to $120 \mathrm{mM} \mathrm{NaCl}$ resulted in a more pronounced increment of WUE during the first two days, followed by a moderate, but significant decline until the fourth day. Priming with SMM enhanced the effect of salt treatment on the increment of water use efficiency at day 2 after starting the salt stress, but this enhancement was reduced on the fourth day of treatment (Fig. 5).

\section{Membrane lipid peroxidation}

Thiobarbituric acid-reactive substances (TBARS) content of canola leaves was not modified by pre-treatment with 1 $\mathrm{m} M$ SMM, but it was significantly increased by salt stress, the highest values being recorded in plants exposed to $120 \mathrm{mM} \mathrm{NaCl}$. Priming with SMM substantially reduced TBARS formation in salt stressed canola: in plants exposed to $60 \mathrm{mM} \mathrm{NaCl}$, SMM pre-treatment completely annihilated the effects of moderate salinity on membrane lipid peroxidation, while in plants treated with $120 \mathrm{~m} M \mathrm{NaCl}$, priming with SMM reduced peroxidative membrane damage to the level of $60 \mathrm{mM} \mathrm{NaCl}$-treated plants without priming (Fig. 6).

\section{Discussion}

Germination is a crucial developmental phase for the onset of new plants, during which young plantlets are especially sensitive to adverse environmental conditions. Salt stress slows down seed germination and results in erratic stand establishment, leading to very low agricultural productivity (Abdolahi et al. 2012; Mahboob et al. 2019). The main inhibitory effect is due to the osmotic component of salt stress, which impairs water absorption needed to start the 


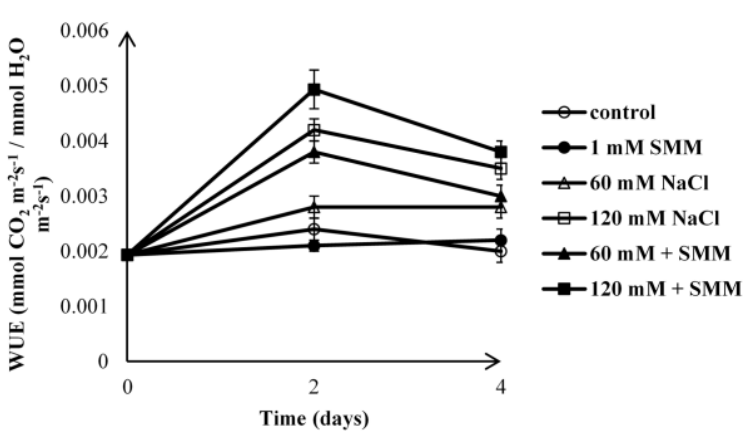

Fig. 5: Effects of two concentrations (60 $\mathrm{m} M$ and $120 \mathrm{~m} M)$ of $\mathrm{NaCl}$ and/or priming with S-methylmethionine (SMM) on the water use efficiency (WUE) of canola leaves, calculated from stomatal gas exchange parameters. Vertical bars represent $\pm \mathrm{SE}$ from means $(n=10)$

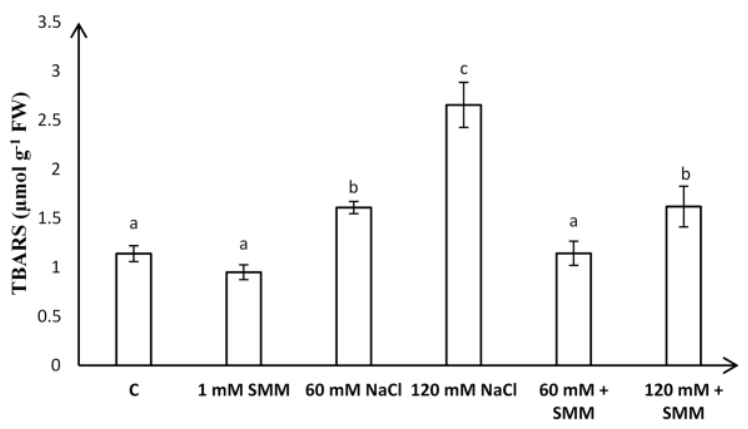

Fig. 6: Membrane lipid peroxidation assayed with the amount of thiobarbituric acid-reactive substances (TBARS) in leaves of canola plants exposed for four days to salinity, with or without priming with S-methylmethionine (SMM). C - control, FW fresh weight

active growth and organogenesis. Seed priming with presoaking for $12 \mathrm{~h}$ in aqueous solution of $1 \mathrm{mM} \mathrm{S}$ methylmethionine helped to maintain a significantly higher germination percentage and reduced the time needed for germination in salt stressed canola seed stands, thus improving salinity tolerance through better emergence. This may be a simple and effective way to overcome the negative impacts of high soil salinity on germination and on the vigor of new plantlets (Tabasssum et al. 2017). Similar results were reported for lettuce seeds, in which cold tolerance during germination and seedling establishment was enhanced with pre-treatment using $0.25 \mathrm{~m} M$ and $2.0 \mathrm{~m} M$ SMM (Fodorpataki et al. 2019). Seed priming was also achieved with a sequential pre-treatment with triacontanol and ascorbate, resulting in improved germination index and reduced germination time of wheat grains exposed to salt stress (Mahboob et al. 2019). They also demonstrated that osmoprotectants' accumulation was higher in primed seeds exposed to salt stress, thus osmoregulation may proceed more efficiently during germination in the presence of high salt concentrations. For canola seeds, priming for $12 \mathrm{~h}$ with $\mathrm{KH}_{2} \mathrm{PO}_{4}(-0.625 \mathrm{MPa})$ and $\mathrm{CaCl}_{2}(-1.25 \mathrm{MPa})$ resulted in vigor enhancement, internal biological processes necessary for germination being intensified by priming (Abdolahi $e t$ al. 2012). Seed soaking for $24 \mathrm{~h}$ before germination with solutions containing thiamin (vitamin $\mathrm{B}_{1}$ ) promoted seedling growth in maize cultivars exposed to salt stress (Kaya et al. 2015). All these results suggest that seed priming may alleviate inhibitory effects of salt stress during germination, pre-treatment being necessary only once and for a short period of time, which makes this procedure cost-effective (Mahboob et al. 2019).

Photosynthetic efficiency decrease under $\mathrm{NaCl}$ indicates that moderate and heavy salt stress during an exposure period of four days disturbs photochemical processes in PSII and reduces the efficiency of light energy conversion into chemical energy in leaves of young canola plants grown under constant light and temperature regimes. Similar results were reported by Zhang et al. (2009) for cucumber seedlings, as well as by Kalaji et al. (2018) for linden. The fact that priming with S-methylmethionine had no effect on ФPSII in plants not exposed to high salinity, but significantly alleviated the reduction of TPSII in saltstressed canola plants (Fig. 2), suggests that this priming agent does not have a direct influence on the functioning of PSII under normal conditions, but it contributes to upregulatory defense mechanisms which result in an improved light use capacity under salt stress conditions. Demetriou $e t$ al. (2007) reported that the protective role of polyamines in the photosynthetic apparatus increases the efficiency of PSII photochemistry in salt-stressed plants. Another photochemical quenching parameter, the efficiency of excitation energy captured by PSII (Fv'/Fm') of canola plants exposed to salt stress was significantly reduced, this reduction being intensified with time, especially in the case of treatment with $120 \mathrm{mM} \mathrm{NaCl}$ (Fig. 3a). Priming with SMM alleviated this reduction, allowing the salt-stressed plants to convert a higher proportion of the absorbed photons into chemical energy which can be subsequently used for carbon dioxide assimilation. Because the efficiency of PSII photochemistry in leaves of salt-stressed canola plants primed with SMM is higher than without SMM pretreatment, one can conclude that SMM improved the photosynthetic capacity of canola plants by increasing the level of photochemical efficiency of PSII under salt stress conditions. Likely, when cucumber seedlings exposed for eight days to $65 \mathrm{mM} \mathrm{NaCl}$ were simultaneously treated with $10 \mathrm{~m} M$ putrescine, the exogenous polyamine supply alleviated the reduction of the efficiency of excitation energy captured by open PSII (Zhang et al. 2009). The fact that exposure of canola plants to $120 \mathrm{mM} \mathrm{NaCl}$ significantly increased NPQ (Fig. 3b) reflects that under the given light intensity, salt stress reduced the photochemical efficiency of PSII, thus a bigger proportion of the absorbed light energy was dissipated as heat in order to avoid subsequent photooxidative damage to the photosynthetic apparatus (Ruban 2016). Under conditions of moderate salt stress (60 $\mathrm{m} M \mathrm{NaCl}$ ) the increase of NPQ is more pronounced after 
two days of salt treatment. In SMM-primed canola plants the increment of NPQ was significantly lower than in nonprimed plants, suggesting the protective role of SMM against functional damages induced by salt stress. Thus, Smethylmethionine enhanced salt tolerance of canola by protecting the photosynthetic apparatus of the thylakoid membranes due to photochemical quenching processes, the efficiency of this preventive protection being associated with a moderated increase in heat dissipation. A similar conclusion was drawn by Poulson et al. (2006), when they primed with UV-B radiation Arabidopsis thaliana plants which were subsequently exposed to drought stress.

Net carbon dioxide uptake through stomatal gas exchange was shown to be related directly to the rate of photosynthetic assimilation. Because stomatal closure is a general defense mechanism under osmotic stress (caused mainly by drought, extreme temperatures and high salinity), less carbon is assimilated under these adverse conditions. This is the main reason why a greater part of the light energy captured by photosynthetic pigments is not used photochemically, and has to be dissipated or induces photooxidative damages which have to be repaired with extra energy investment (Farooq et al. 2013; Ceusters et al. 2019). The present study showed that salt stress decreases net photosynthetic carbon assimilation rate (Pn) proportionally with the $\mathrm{NaCl}$ concentration, and mainly during the first days of exposure (Fig. 3c). This may be a result of stomatal closure as a defense mechanism against osmotic stress induced by high salinity. Stomatal closure impairs net carbon dioxide uptake, which results in a decreased rate of photosynthetic assimilation (Ceusters et al. 2019). The fact that $\mathrm{Pn}$ of plants without $\mathrm{NaCl}$ showed no influence by SMM, but priming with SMM resulted in a more moderated reduction of this rate in salt-stress plants, suggests that this substance enables canola plants to cope more successfully with disturbances caused by high salinity in the photosynthetic process. Because alleviation of salt stress by pre-treatment with $1 \mathrm{~m} M$ SMM was less pronounced in the case of stomatal conductance for water vapor and transpiration rate than in the case net carbon assimilation rate (data not shown), one may conclude that SMM is involved in the improvement of photosynthetic capacity in a higher extent by increasing the level of the photochemical efficiency than by regulation of stomatal movements. This was also found for putrescine in $\mathrm{NaCl}-$ stressed cucumber seedlings, but in the latter case this polyamine had no effect at all on stomatal conductance and on transpiration rate of leaves (Zhang et al. 2009). On the other hand, priming with UV-B radiation had a much greater influence on transpiration rate than on net carbon assimilation rate when Arabidopsis thaliana plants were exposed to increasing light intensities (Poulson et al. 2006). In wheat, high salinity $(150 \mathrm{~m} M \mathrm{NaCl})$ caused a reduction in all gas exchange parameters, while exogenous ascorbic acid induced a similar increase in stomatal conductance for water vapor and in the carbon dioxide assimilation rate of both salt-stressed and non-stressed plants (Farooq et al. 2013). These results show that different priming agents may have disparate effects on various parameters of stomatal gas exchange under several stress conditions.

The fact that in absence of salt stress leaf spraying with SMM causes a moderate, but statistically significant increase of the chlorophylls to carotenoids ratio (due to a higher total chlorophyll content - data not shown) may be related to the fact that, as an amino acid derivative, SMM stimulates the biosynthesis of chlorophyll. Sadak et al. (2015) reported that foliar spraying of a mixture of amino acids increased the chlorophyll content of faba bean leaves. Zhang et al. (2009) reported an increment of chlorophyll content in leaves of cucumber plants exposed to salt stress, with a tendency of reduction at day 8 after the start of salt treatment. Intense UV-B radiation was also shown to induce an increased photosynthetic pigment content of Arabidopsis leaves. In several experiments, carotenoid pigment content increased when stress factors impaired the use of light energy in carbon assimilation and a higher amount of the absorbed light energy had to be dissipated in order to prevent extensive photooxidative damage. This situation may occur under salt stress, and the increment of photoprotective carotenoid pigment content may explain the reduction of chlorophylls to carotenoids ratio in the canola leaves exposed to high salinity. On the other hand, the fact that priming with $1 \mathrm{~m} M$ SMM totally compensated for this influence of salt stress and in plants grown in the presence of $60 \mathrm{mM} \mathrm{NaCl}$ even increased the value of this pigment ratio above the value which was characteristic for the control plants, suggests that SMM may participate in the acclimation of the photosynthetic apparatus to high salinity conditions and may contribute to the protection of thylakoid membranes against oxidative damage. This correlates with the results which show that SMM pretreatment reduces membrane lipid peroxidation in plants exposed to salinity stress. A similar role of putrescine was demonstrated in cucumber seedlings exposed to salt stress induced by a treatment for 8 days with $65 \mathrm{mM} \mathrm{NaCl}$ (Zhang et al. 2009).

Pre-treatment with $1 \mathrm{~m} M$ SMM had no influence on WUE, while in two days after the onset of salt stress a significant increase of water use efficiency indicates that the osmotic component of high salinity induces regulation of stomatal conductance to water vapor and of carbon dioxide assimilation, which results in increased drought tolerance. Transpiration rate is lowered by salt stress more intensely than net carbon dioxide assimilation, thus water use efficiency increases. This increment is intensified if saltstressed plants were primed with SMM, the enhancement being reduced on the fourth day of salt treatment. Because SMM alleviated the inhibitory effect of salt stress on net photosynthetic carbon assimilation, but exerted a weaker influence on transpiration rate significantly reduced by salt stress due to stomatal closure (data not shown), one can conclude that S-methylmethionine affects the 
photosynthetic carbon assimilation rather than stomatal movements. Partly similar results were obtained when saltstressed cucumber seedlings treated with $10 \mathrm{~m} M$ putrescine exhibited an enhanced water use efficiency at day 1 after the beginning of salt treatment, but during the next days the enhancement was gradually reduced (Zhang et al. 2009). This was related with the fact that putrescine moderated the reduction of net photosynthetic rate by salt stress but had no significant influence on stomatal conductance and transpiration rate. In wheat, salt stress induced with $150 \mathrm{mM}$ $\mathrm{NaCl}$ decreased water use efficiency because of a more intense reduction of carbon dioxide assimilation rate than that of transpiration rate, while priming with $100 \mathrm{mg} \mathrm{L}^{-1}$ ascorbic acid (vitamin $\mathrm{C}$, applied to the rooting medium) caused an increase in WUE of non-stressed plants, but had no significant influence on WUE of salt-stressed plants (Farooq et al. 2013).

In the present study it was found that exogenous application of $1 \mathrm{~m} M$ SMM before the exposure of young canola plants to moderate and severe salinity stress mitigated the effect of salt stress on membrane damage reflected by significant accumulation of TBARS. This can be associated with reduced generation of reactive oxygen species in SMM-treated canola plants subsequently exposed to salt stress. It is suggested that increase in antioxidative activities in salt-stressed plants is an additional burden on metabolism which may be reduced by priming with SMM. Similar results were reported by Kaya et al. (2015) for saltstressed maize cultivars, when exogenous application of thiamine reduced hydrogen peroxide and malondialdehyde formation by modulating the cellular redox status and the activity of antioxidant enzymes, resulting in a reduced metabolic cost of stress tolerance. Pre-treatment with 100 $\mathrm{m} M$ ascorbic acid also reduced membrane lipid peroxidation in pepino plantlets exposed to chilling stress, and its beneficial influence on stress tolerance was related to the fact that it modulated the activities of antioxidant enzymes involved in membrane protection (Sivaci et al. 2014). The current results support the idea that priming with millimolar amounts of S-methylmethionine as foliar spray may efficiently prevent oxidative membrane damage induced by salt stress in the leaves of canola plants. However, the role of exogenously applied SMM in the antioxidative defense system of plants yet needs to be elucidated.

\section{Conclusion}

Priming with S-methylmethionine can be effectively used to enhance salinity tolerance of canola during its early developmental stages. It compensates for the delayed germination under salt stress, restores photosynthetic performance under moderate salt stress and alleviates its reduction by severe salt stress. Reduction of chlorophylls to carotenoids ratio and increment of membrane lipid peroxidation in leaves are annihilated by pre-treatment with SMM as a foliar spray, while water use efficiency is improved under high salinity. The current results open a perspective for metabolic engineering aiming an enhanced production of SMM by the canola plant itself, to achieve an increased production under adverse cultivation conditions. From a crop improvement perspective, modulation of SMM metabolism using its exogenous application may also be an innovative way to improve abiotic stress tolerance in several crop plants.

\section{Acknowledgements}

We thank for the support given to Ph.D. student Katalin Molnar by the doctoral school of the University of Agricultural Sciences and Veterinary Medicine in ClujNapoca, and to M.Sc. student Bernat Tompa by the Institute of Advanced Studies in Science and Technology of the "Babes-Bolyai" University in Cluj-Napoca, Romania.

\section{Author Contributions}

Laszlo Fodorpataki: planning and supervision of the work, methodology and editing. Katalin Molnar: methodology and data analysis. Bernat Tompa: methodology and data analysis. Csaba Bartha: methodology, data analysis, review and editing.

\section{References}

Abdolahi M, B Andelibi, E Zangani, F Shekari, SJ Somarin (2012). Effect of accelerated aging and priming on seed germination of rapeseed (Brassica napus L.) cultivars. Intl Res J Appl Basic Sci 3:499-508

Antoniou C, A Savvides, A Christou, V Fotopoulos (2016). Unravelling chemical priming machinery in plants: The role of reactive oxygennitrogen-sulfur species in abiotic stress tolerance enhancement. Curr Opin Plant Biol 33:101-107

Bajwa AA, M Farooq, A Nawaz (2018). Seed priming with sorghum extracts and benzyl aminopurine improves the tolerance against salt stress in wheat (Triticum aestivum L.). Physiol Mol Biol Plants 24:239-249

Balestrini R, W Chitarra, C Antoniou, R Ruocco, V Fotopoulos (2018). Improvement of plant performance under water deficit with the employment of biological and chemical priming agents. J Agric Sci 156:680-688

Baryla A, C Laborde, JL Montillet, C Triantaphylides, P Chagvardieff (2000). Evaluation of lipid peroxidation as a toxicity bioassay for plants exposed to copper. Environ Pollut 109:131-135

Blumwald E (2000). Sodium transport and salt tolerance in plants. Curr Opin Cell Biol 12:431-434

Ceusters N, R Valcke, M Frans, JE Claes, W Erde, J Ceusters (2019). Performance index and PSII connectivity under drought and contrasting light regimes in the CAM orchid Phalaenopsis. Front Plant Sci 10; Article 1012

Demetriou G, C Neonaki, E Navakoudis, K Kotzabasis (2007). Salt stress impact on the molecular structure and function of the photosynthetic apparatus - the protective role of polyamines. Biochim Biophys Acta 1767:272-280

Downton WJS, BR Loveys, WJR Grant (1990). Salinity effects on the stomatal behavior of grapevine. New Phytol 116:499-503

Farooq M, A Nawaz, MAM Chaudhry, R Indrasti, A Rehman (2017). Improving resistance against terminal drought in bread wheat by exogenous application of proline and gamma-aminobutyric acid. $J$ Agron Crop Sci 203: 464-472

Farooq M, A Ullah, DJ Lee, SS Alghamdi (2018) Terminal droughtpriming improves the drought tolerance in Desi and Kabuli chickpea. Intl J Agric Biol 20:1129-1136 
Farooq M, M Irfan, T Aziz, I Ahmad, SA Cheema (2013) Seed priming with ascorbic acid improves drought resistance of wheat. J Agron Crop Sci 199:12-22

Farooq M, M Usman, F Nadeem, H Rehman, A Wahid, SMA Basra, KHM Siddique (2019) Seed priming in field crops - potential benefits, adoption and challenges. Crop Pasture Sci 70:731-771

Farooq M, SMA Basra, A Wahid, H Rehman (2009) Exogenously applied nitric oxide enhances the drought tolerance in fine grain aromatic rice (Oryza sativa L.). J Agron Crop Sci 195:254-261

Fiebelkorn D, M Rahman (2016). Development of a protocol for frosttolerance evaluation in rapeseed/canola (Brassica napus L.). Crop J 4:147-152

Fodorpataki L, K Molnar, B Tompa, SRC Plugaru (2019). Priming with vitamin $\mathrm{U}$ enhances cold tolerance of lettuce (Lactuca sativa L.). Not Bot Hortic Agrobot 47:592-598

Fodorpataki L, B Holinka, E Gyorgy (2016). Priming with Smethylmethionine increases non-enzymatic antioxidant content of lettuce leaves exposed to salt stress. In: Controlled Environment Agriculture, pp:133-164. Asaduzzaman M (Ed.). Nova Science Publishers, New York, USA

Hoagland DR, DI Arnon (1950). The water-culture method for growing plants without soil. Calif Agric Exp Stat Circ 347:1-32

Hollington PA, KA Steele (2007). Participatory breeding for drought and salt tolerant crops. In: Advances in Molecular Breeding toward Drought and Salt Tolerant Crops, pp:455-478. Jenks, MA, PM Hasegawa, SM Jain (Eds.). Springer, Dordrecht, The Netherlands

Iqbal M, R Khan, M Asgher, N Iqbal, NA Khan (2013). Potentiality of sulphur-containing compounds in salt stress tolerance. In: Ecophysiology and Responses of Plants under Salt Stress, pp:443472. Ahmad P, MM Azooz, MNV Prasad (Eds.). Springer, New York, USA

Jambunathan N (2010). Determination and detection of reactive oxygen species (ROS), lipid peroxidation, and electrolyte leakage in plants; pp:219-297. In: Plant Stress Tolerance. Methods in Molecular Biology (Methods and Protocols), vol 639. Sunkar R. (Ed.). Humana Press, New Jersey, USA

Kalaji HM, L Rackova, V Paganova, T Swoczyna, S Rusinowski, K Sitko (2018). Can chlorophyll-a fluorescence parameters be used as bioindicators to distinguish between drought and salinity stress in Tilia cordata Mill.? Environ Exp Bot 152:149-157

Kaya C, M Ashraf, O Sonmez, AL Tuna, T Polat, S Aydemir (2015). Exogenous application of thiamin promotes growth and antioxidative defense system at initial phases of development in salt-stressed plants of two maize cultivars differing in salinity tolerance. Acta Physiol Plantarum 37:1729-1741

Kooten O, J Snel (1990). The use of chlorophyll fluorescence nomenclature in plant stress physiology. Photosynth Res 25:147-150

Mahboob W, MA Khan, MU Shirazi, S Faisal, A Asma (2019). Seed priming modulates germination potential, osmoprotectants accumulation and ionic uptake in wheat seedlings under salt stress. Intl J Agric Biol 22:594-600

Maxwell K, GN Johnson (2000). Chlorophyll fluorescence - a practical guide. J Exp Bot 51:659-668

McRorie RA, GL Sutherland, G Lewis, MS Barton, MR Glazener (1954). Isolation and identification of a naturally occurring analog of methionine. J Amer Chem Soc 76:115-118

Nayidu N, V Bollina, S Kagale (2013). Oilseed crop productivity under salt stress. In: Ecophysiology and Responses of Plants under Salt Stress, pp:249-266. Ahmad P, MM Azooz, MNV Prasad (Eds.). Springer, New York, USA

Nieva FJJ, EM Castellanos, ME Figueroa, F Gil (1999). Gas exchange and chlorophyll fluorescence of $\mathrm{C}_{3}$ and $\mathrm{C}_{4}$ saltmarsh species. Photosynthetica 36:397-406

Paldi K, IRacz,ZSzigeti, S Rudnoy (2014). S-methylmethionine alleviates the cold stress by protection of the photosynthetic apparatus and stimulation of the phenylpropanoid pathway. Biol Plantarum 58:189-194
Patel AD, NK Prajapati (2012). Review on biochemical importance of vitamin-U. J Chem Pharm Res 4:209-215

Poulson ME, MRT Boeger, RA Donahue (2006). Response of photosynthesis to high light and drought for Arabidopsis thaliana grown under a UV-B enhanced light regime. Photosynth Res 90:7990

Ranocha P, SD McNeil, MJ Ziemak, C Li, MC Tarczynski, AD Hanson (2001). The S-methylmethionine cycle in angiosperms: Ubiquity, antiquity and activity. Plant $J$ 25:575-584

Rasheed R, MA Ashraf, S Parveen, M Iqbal, I Hussain (2014). Effect of salt stress on different growth and biochemical attributes of two canola (Brassica napus L.) cultivars. Commun Soil Sci Plant Anal 45:669679

Rehman A, M Farooq, M Naved, A Nawaz, B Shahzad (2018) Seed priming of $\mathrm{Zn}$ with endophytic bacteria improves the productivity and grain biofortification of bread wheat. Eur J Agron 94:98-107

R Core Team (2019). R: A Language and Environment for Statistical Computing. R Foundation for Statistical Computing, Vienna, Austria

Ruban AV (2016). Nonphotochemical chlorophyll fluorescence quenching: Mechanism and effectiveness in protecting plants from photodamage. Plant Physiol 170:1903-1916

Sadak MSH, MT Abdelhamid, U Schmidhalter (2015). Effects of foliar application of amino acids on plant yield and some physiological parameters in faba bean plants irrigated with seawater. Acta Biol Colomb 20:141-152

Savvides A, S Ali, M Tester, V Fotopoulos (2016). Chemical priming of plants against multiple abiotic stresses: Mission possible? Trends Plant Sci 21:329-340

Shabhaz M, N Noreena, S Perveen (2013). Triacontanol modulates photosynthesis and osmoprotectants in canola (Brassica napus L.) under saline stress. J Plant Interact 8:350-359

Sivaci A, A Kaya, S Duman (2014). Effects of ascorbic acid on some physiological changes of pepino (Solanum muricatum Ait.) under chilling stress. Acta Biol Hung 65:305-318

Srivastava AK, S Srivastava, HV Lokhande, SFD Souza, P Suprasanna (2015). Salt stress reveals differential antioxidant and energetic responses in glycophyte (Brassica juncea L.) and halophyte (Sesuvium portulacastrum L.). Front Environ Sci 3; Article 19

Tabasssum T, M Farooq, R Ahmad, A Wahid (2017). Seed priming and transgenerational drought memory improves tolerance against salt stress in bread wheat. Plant Physiol Biochem 18:362-369

Tang W, RJ Newton (2005). Polyamines reduce salt-induced oxidative damage by increasing the activities of antioxidant enzymes and decreasing lipid peroxidation in Virginia pine. Plant Growth Regul 46:31-43

Vannier N, C Mony, AK Bittebiere, P Vandenkoornhuyse (2015). Epigenetic mechanisms and microbiota as a toolbox for plant phenotypic adjustment to environment. Front Plant Sci 6; Article 1159

Wellburn AR (1994). The spectral determination of chlorophylls $a$ and $b$, as well as total carotenoids, using various solvents with spectrophotometers of different resolution. J Plant Physiol 144:307313

Xia JR, YJ Li, DH Zou (2004). Effects of salinity stress on PSII in Ulva lactuca as probed by chlorophyll fluorescence measurements. Aquat Bot 80:129-137

Yang $\mathrm{XH}, \mathrm{CM} \mathrm{Lu}$ (2005). Photosynthesis is improved by exogenous glycinebetaine in salt-stressed maize plants. Physiol Plantarum 124:343-352

Zamani S, A Bybordi, MB Khorshidi, T Nezami (2010). Effect of $\mathrm{NaCl}$ salinity levels on lipids and proteins of canola (Brassica napus L.) cultivars. Adv Environ Biol 4:397-403

Zhang RH, J Lin, SR Guo, T Tezuka (2009). Effects of exogenous putrescine on gas-exchange characteristics and chlorophyll fluorescence of $\mathrm{NaCl}$-stressed cucumber seedlings. Photosynth Res 100:155-162

Zhu J (2001). Plant salt tolerance. Trends Plant Sci 6:66-72 